\title{
Investigation into the utility of an immunocytochemical assay in body cavity effusions for diagnosis of feline infectious peritonitis
}

Journal of Feline Medicine and Surgery 2017. Vol. 19(4) 410-418 (C) The Author(s) 2016 Reprints and permissions: sagepub.co.uk/journalsPermissions.nav DOI: 10.1177/1098612X16630357 journals.sagepub.com/home/jfms

(S)AGE

\section{Sandra Felten', Kaspar Matiasek2, Stefanie Gruendl1, Laura Sangl', Gerhard Wess ${ }^{1}$ and Katrin Hartmann' ${ }^{1}$}

\begin{abstract}
Objectives Feline coronaviruses (FCoVs) exist as two biotypes, feline enteric coronavirus and feline infectious peritonitis virus. Although feline infectious peritonitis (FIP) is a very common disease, the ante-mortem diagnosis of this disease still remains a challenge. Immunofluorescence staining of FCoV in macrophages in effusion has been considered as the reference standard for the diagnosis, but recently this method has been shown to have lower specificity than previously reported. In addition, this method is not widely available and requires the use of fluorescence microscopes. Therefore, it was the aim of this study to evaluate the diagnostic potential of an immunocytochemical (ICC) assay using body cavity effusion.

Methods Effusion samples from 27 cats with immunohistochemically confirmed FIP and 29 cats with suspected FIP but a definitive diagnosis of another disease were examined. ICC specimens were evaluated with respect to positive immunostaining. In addition, effusion samples were stained with haematoxylin and eosin and evaluated cytologically.

Results A diagnostic sensitivity of $85.2 \%$ was recorded for effusion specimens (95\% confidence interval [Cl] 66.3-95.8), while the diagnostic specificity was only $72.4 \%$ (95\% Cl 52.8-87.3).

Conclusions and relevance Once the clinical disease of FIP develops in a cat, it always leads to death, and most of the cats are euthanased within a few days or weeks. As false-positive results might lead to euthanasia of cats suffering from potentially treatable diseases, the diagnostic specificity of a diagnostic tool is the most important factor in a fatal disease like FIP. Thus, the diagnostic utility of this test proved to be insufficient and positive ICC results should be interpreted with caution. Nevertheless, full-body necropsy could not be performed in 13/29 control cats. It is possible that these cats actually suffered from early-stage FIP and that this fact might have influenced the diagnostic specificity of the ICC. Based on the results of the present study, however, ICC of effusion samples currently cannot be recommended to confirm a suspicion of FIP.
\end{abstract}

Accepted: 8 January 2016

\section{Introduction}

Feline infectious peritonitis (FIP) is one of the leading infectious causes of death among the cat population worldwide. The causative agent of FIP is the feline coronavirus (FCoV) that occurs as two distinct biotypes, feline enteric coronavirus (FECV) and feline infectious peritonitis virus (FIPV). ${ }^{1-3}$ Coronaviruses are well known for their high mutability.4-6 The internal mutation hypothesis, which is widely accepted, suggests that FIPV originates as a mutant from its parental FECV within an infected cat.7,8 Both FECV and FIPV
${ }^{1}$ Clinic of Small Animal Medicine, Centre for Clinical Veterinary Medicine, Ludwig-Maximilians-University Munich, Munich, Germany

2Section of Clinical and Comparative Neuropathology, Institute of Veterinary Pathology, Centre for Clinical Veterinary Medicine, Ludwig-Maximilians-University Munich, Munich, Germany

\section{Corresponding author:}

Sandra Felten, Clinic of Small Animal Medicine, Centre for Clinical Veterinary Medicine, Ludwig-Maximilians-University Munich,

Veterinaerstrasse 13, 80539 Munich, Germany

Email: s.felten@medizinische-kleintierklinik.de 
infect macrophages, but only the disease-causing FIPV is capable of efficiently replicating within the macrophages and maintaining the replication at a sufficient level. ${ }^{9,10}$ The replication of the virus within the macrophages is believed to play the central role in the development of FIP. ${ }^{11}$ Replication leads to a positive immunostaining of viral antigen in the cytoplasm of macrophages. ${ }^{12}$

If a cat is presented with effusion, a number of diagnostic tests can be performed. However, macroscopic, cytological and biochemical analysis of effusion fluid can be misleading, as abnormalities are not specific for FIP. ${ }^{13,14}$ The Rivalta test is a simple and inexpensive test, which has been used to differentiate transudates from exudates. ${ }^{15}$ This test has been frequently used in feline effusions, but it can produce false-positive results. ${ }^{16}$ Reverse transcriptase PCR and measurement of FCoV antibodies in effusion, although more promising than in blood, ${ }^{17-19}$ are not diagnostic and should only be interpreted in conjunction with history, clinical presentation and results of other tests. ${ }^{12,20,21}$ Therefore, the diagnosis of FIP remains a challenge, even in cats with body cavity effusion.

In earlier studies evaluating the utility of immunostaining of FCoV antigen within macrophages, immunofluorescence was used in effusion samples. ${ }^{17,22-25}$ This technique was shown to have excellent specificity for the diagnosis of FIP in earlier studies; ${ }^{17,23-25}$ however, most of these studies were retrospective in nature and lacked necropsy confirmation of FIP vs other diseases. In contrast, a recent study revealed false-positive results of an immunofluorescence assay in two cats with diseases other than FIP. ${ }^{22}$ In contrast to immunofluorescence, immunocytochemistry (ICC) uses chromogens rather than fluorochromes such as fluorescein isothiocyanate, allowing assessment by standard bright field microscopy and cytomorphological evaluation of the cells. Moreover, this method does not require a fluorescence microscope, which makes the technique accessible to more diagnostic facilities. Additionally, ICC has the advantage of the possibility to reassess the slides at a later point in time, whereas immunofluorescence staining fades with time, and thus allows the slides to be transported to other laboratories for further evaluation.

The aim of this study was to evaluate the sensitivity and specificity of an ICC assay in effusion from cats with confirmed FIP and a defined control group of cats for which FIP was considered an important differential diagnosis by a clinician. It was hypothesised that this technique would allow a definitive ante-mortem diagnosis of FIP in cats with effusion. All samples were additionally evaluated cytologically in order to facilitate the assessment of the ICC results.

\section{Materials and methods}

\section{Animals}

The study was designed as a prospective study and included 56 cats. All the cats were suspected of having FIP based on clinical and/or laboratory signs and were therefore either presented as patients to the Clinic of Small Animal Medicine $(n=41)$, or directly submitted for necropsy to the Institute of Veterinary Pathology, Centre for Clinical Veterinary Medicine, LudwigMaximilian-University, Munich, Germany $(\mathrm{n}=15)$.

All 56 cats were classified into two groups. The FIP group $(n=27)$ consisted of cats definitively diagnosed with FIP in necropsy. The diagnosis was established by histopathology and immunohistochemical staining of FCoV antigen in macrophages in FIP-typical lesions in tissue samples obtained at necropsy. For immunohistochemistry (IHC), formalin-fixed, paraffin-embedded tissue samples were cut into $5 \mu \mathrm{m}$ sections and dried overnight at $37^{\circ} \mathrm{C}$. These sections were then deparaffinised in xylene and rehydrated in a graded series of alcohol. In order to reverse the antigen-masking effects due to fixation, heat-induced antigen retrieval was carried out using microwave pretreatment in citrate buffer ( $\mathrm{pH}$ 6.0). The tissue samples were then further processed as described below for ICC on effusion slides.

The control group $(n=29)$ consisted of cats for which FIP was regarded as a differential diagnosis by clinicians because of the presence of effusion. Cats were only included if a definitive diagnosis of a disease other than FIP, which explained the clinical signs, was established. These diagnoses were confirmed either by full postmortem examination, including histopathology $(n=15)$, by histopathology of organ samples obtained post mortem $(n=1)$, by bacterial culture and cytology to diagnose bacterial pleuritis or peritonitis $(n=2)$, by echocardiography to diagnose decompensated cardiac disease explaining pleural or abdominal effusion $(n=7)$, or by cytology to diagnose neoplasia $(n=4)$. In order to reduce the likelihood of failure to detect FIP as a comorbidity in control cats, the necropsy protocols for the 15 control cats in which full post-mortem examination was performed included IHC for FCoV in the affected tissues. All 15 cats showed negative IHC results.

\section{Samples}

In total, 57 effusion samples were collected between 2012 and 2014. From one cat in the FIP group, two effusion samples (ascites and pleural effusion) were obtained. Effusion samples were collected either ante-mortem by abdominocentesis or thoracocentesis, or post mortem.

Aliquots of $100 \mu \mathrm{l}$ of the effusion samples (ascites, $\mathrm{n}=32$; pleural effusion, $n=23$; pericardial effusion, $n=2$ ) were cytocentrifuged in a cytospin centrifuge (Universal R; Hettich), and the slides were stored at $-20^{\circ} \mathrm{C}$ until use. At 
least two slides of each sample were prepared for each cat. The effusion samples collected ante-mortem were originally obtained for diagnostic and/or therapeutic purposes.

\section{Cytological evaluation}

One slide of each effusion sample was stained with haematoxylin and eosin and evaluated semi-quantitatively for cellularity, cellular composition, blood contamination/ haemorrhage and erythrophagocytosis, protein, aspiration of autochthonous cells (eg, liver cells) and mesothelial cells (Table 1 in supplementary material). Based on these parameters, slides were classified as cytologically 'typical for FIP', 'compatible with FIP' or 'not indicative of FIP'. Pyogranulomatous cytology with engulfment of neutrophils by macrophages and absence of microorganisms and giant cells was regarded as 'typical for FIP' (Figure 1). Mixed white blood cell populations containing macrophages were regarded as 'compatible with FIP'. Other cellular compositions, such as exclusively neutrophilic or neoplastic cells, were regarded as 'not indicative of FIP'.

ICC

Slide reading and interpretation of ICC results were performed by two independent investigators, who were blinded to all data of the cats, including their diagnoses.

The cytological slides for ICC were thawed at room temperature. The slides remained wrapped in aluminium foil during thawing in order to avoid condensation, which could lead to the loss of cellular material on the slides. In order to reduce background staining, endogenous peroxidase was inhibited using $2.3 \%$ hydrogen peroxide in $0.01 \mathrm{M}$ phosphate-buffered saline (PBS; pH 7.0-7.4) for cytological samples and 3\% hydrogen peroxide in methanol for histological samples. After washing these slides three times with PBS, all the slides were incubated with normal goat serum (1:20; MP Biomedicals) in a humidity chamber for 30 mins at room temperature to block non-specific binding of the antibodies. An anti-FCoV mouse monoclonal IgG2A (FIPV3-70; Linaris) diluted to 1:400 in PBS was applied as the primary antibody, and the slides were incubated overnight at $4^{\circ} \mathrm{C}$ in a humidity chamber. The following day, after washing the slides three times with PBS, the secondary antibody, a goat antimouse IgG conjugated to biotin (Dako), diluted to 1:200 in PBS was applied. The slides were incubated in the humidity chamber for $1 \mathrm{~h}$ at room temperature and afterwards washed three times with PBS. A complex of avidin and biotinylated horseradish peroxidase $(A B C)$ (Vectastain $A B C$ Kit; Vector Laboratories) was formed by preincubating the mixture for 30 mins. The slides were then incubated with the $\mathrm{ABC}$ for $1 \mathrm{~h}$ at room temperature in the humidity chamber. The slides were washed three times with PBS followed by the addition of 3,3-diaminobenzidine tetrahydrochloride $(\mathrm{pH}$ 7.0; Kem-En-Tec Diagnostics) acting as chromogen and incubated for 5 mins at room temperature. Then the slides were washed with PBS and dipped in distilled water followed by counter-staining with Mayer's Hemalaun (AppliChem $\mathrm{GmbH}$ ) and rinsing in running tap water. The slides were then dehydrated in an ascending series of alcohol and coverslipped using xylene-based mounting medium (Histokitt; Glaswarenfabrik Karl Hecht GmbH $\&$ Co. KG). In order to ensure adequate performance of the antibody, a positive tissue control was included in each of the staining protocols.

Cellularity and positivity of the immunostained samples were evaluated using bright-field microscopy. Only samples with $>50$ nucleated cells in total were included in the statistical analysis. Samples lacking unimpaired cellular material were excluded from statistical analysis. If positive immunostaining was present within a sample, cells with a positive signal were further assessed considering the cell type, the signal pattern and the intensity of the staining. A positive sample was defined as containing macrophages with brown, intensely coloured and granulated cytoplasm (Figure 1). A negative sample was defined as containing macrophages without any immunostaining or cells with non-specific immunostaining. Immunostaining of mesothelial cells, lymphocytes, plasma cells, neutrophils, or erythrocytes was considered non-specific. Also, a diffuse and / or light staining of the cytoplasm was considered non-specific. Non-specific staining was considered negative in ICC. All ICCpositive samples were categorised semi-quantitatively with respect to their yield of true positive cells (low-yield positive: few single macrophages with strong positive signal; medium-yield positive: about half of the macrophages with strong positive signal; high-yield positive: majority of macrophages with strong positive signal).

\section{Statistical evaluation}

To evaluate the diagnostic value of the ICC assay in the diagnosis of FIP, sensitivity (the proportion of positive test results among all cats with FIP), specificity (the proportion of negative test results among all cats with diseases other than FIP), positive predictive value (PPV; the probability that a cat with a positive test result has FIP), negative predictive value (NPV; the probability that a cat with a negative test result does not have FIP) and overall accuracy (the sum of true positive and true negative test results divided by the total number of test results) were determined. To quantify uncertainty, $95 \%$ confidence intervals (CIs) were calculated. Statistical analysis was performed using Microsoft Excel and Prism version 5.04 (GraphPad Software). 


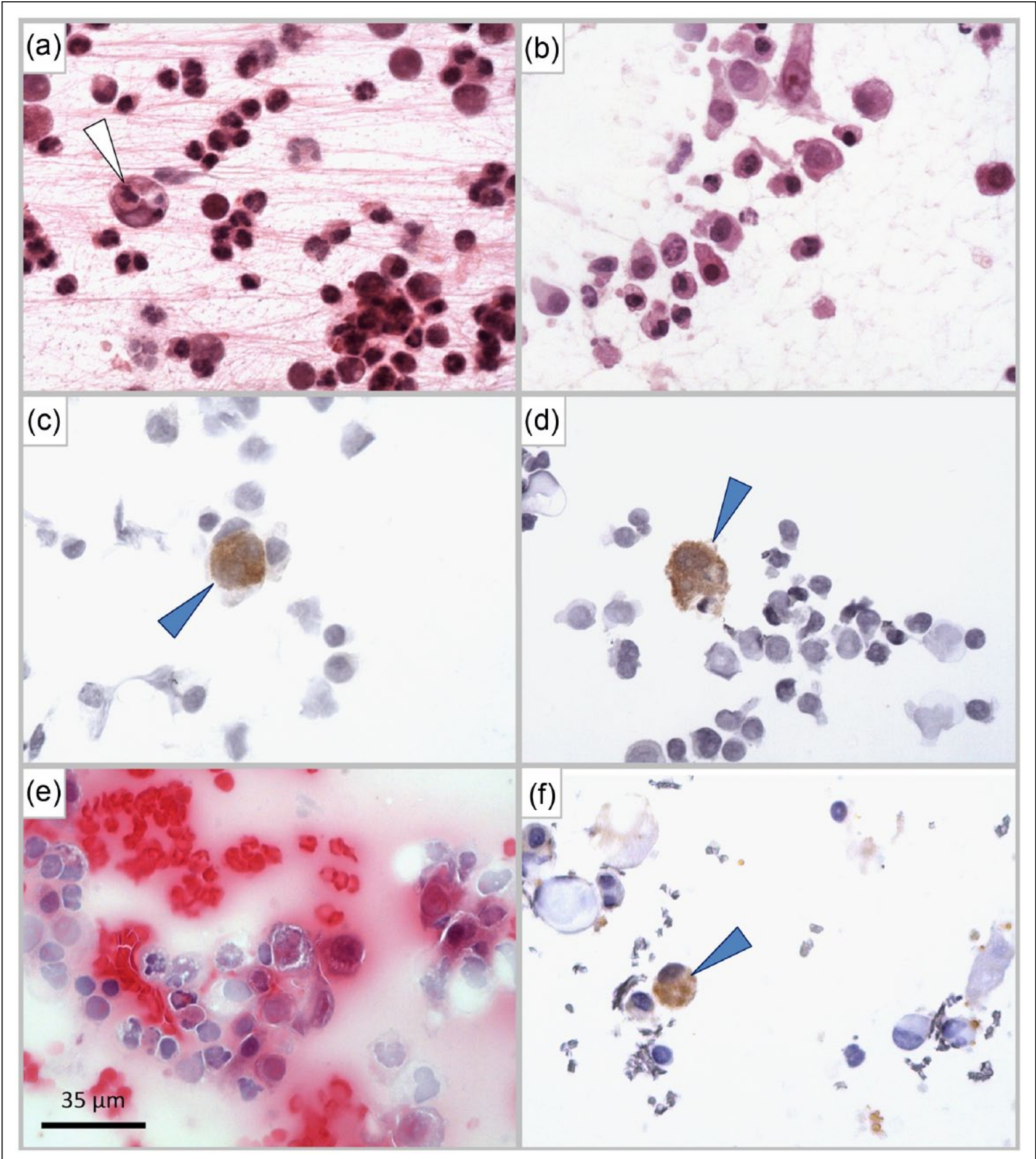

Figure 1 Cytological and immunocytological findings. (a) Feline infectious peritonitis (FIP)-typical cytology showing macrophages engulfing polymorphonuclear neutrophils (arrowhead) and extracellular fibrin mesh. (b) Cytology compatible with FIP, seen in cats with other inflammatory aetiologies. Immunopositive macrophages (arrowheads) seen in (c) peritoneal effusion and (d) pleural effusion. (e,f) FIP-compatible cytology; (e) with non-specific immunopositive cells and (f) within the effusion of a control cat affected by purulent and granulomatous epi- and myocarditis

\section{Results}

Cytological evaluation

The results of the cytological evaluation of effusion samples are presented in Table 1. There was a good to very good intra- and inter-rater agreement throughout. Overall, the cellular composition was either predominantly neutrophilic with $(n=1)$ or without necrotic cells $(n=6)$, predominantly lymphocytic-monocytic $(n=7)$, 
Table 1 Cytological evaluation of 57 haematoxylin and eosin-stained effusion samples from the 56 cats (for definitions see supplementary material)

\begin{tabular}{|c|c|c|c|}
\hline \multicolumn{2}{|l|}{ Group } & \multirow{2}{*}{$\begin{array}{l}\text { FIP } \\
28\end{array}$} & \multirow{2}{*}{$\begin{array}{l}\text { Controls } \\
29\end{array}$} \\
\hline Number of samples & & & \\
\hline Cytological signs for FIP & Typical & 19 & 4 \\
\hline & Compatible & 5 & 5 \\
\hline & Not indicative & 4 & 20 \\
\hline \multirow[t]{3}{*}{ Cellularity } & Low & 9 & 9 \\
\hline & Medium & 13 & 10 \\
\hline & High & 6 & 10 \\
\hline \multirow{4}{*}{$\begin{array}{l}\text { Blood contamination/ } \\
\text { haemorrhage }\end{array}$} & Negative & 6 & 2 \\
\hline & + & 11 & 6 \\
\hline & ++ & 10 & 9 \\
\hline & +++ & 1 & 12 \\
\hline \multirow{4}{*}{ Erythrophagocytosis } & Negative & 11 & 8 \\
\hline & + & 13 & 15 \\
\hline & ++ & 4 & 5 \\
\hline & +++ & 0 & 1 \\
\hline \multirow[t]{4}{*}{ Protein } & Negative & 3 & 5 \\
\hline & + & 10 & 11 \\
\hline & ++ & 10 & 11 \\
\hline & +++ & 5 & 2 \\
\hline \multirow[t]{4}{*}{ Fibrin } & Negative & 9 & 15 \\
\hline & + & 8 & 9 \\
\hline & ++ & 8 & 1 \\
\hline & +++ & 3 & 4 \\
\hline \multirow{4}{*}{$\begin{array}{l}\text { Aspiration of autochthonous } \\
\text { cells }\end{array}$} & Negative & 20 & 23 \\
\hline & + & 5 & 2 \\
\hline & ++ & 3 & 3 \\
\hline & +++ & 0 & 1 \\
\hline \multirow[t]{5}{*}{ Mesothelial cells } & Negative & 4 & 7 \\
\hline & + & 11 & 12 \\
\hline & ++ & 12 & 7 \\
\hline & +++ & 1 & 1 \\
\hline & Not determined & 0 & 2 \\
\hline
\end{tabular}

FIP = feline infectious peritonitis

predominantly lymphocytic $(\mathrm{n}=3)$, pyogranulomatous with $(n=2)$ or without necrotic cells $(n=22)$, or neoplastic $(n=7)$. A mixed leukocyte population was present in five of the effusion samples. In the remaining four effusion samples, pleocytosis was absent.

\section{ICC}

Cellularity and the results of the immunostaining of all samples from cats with FIP are presented in Table 2. The results of the control cats are presented in Table 3. Of the 57 effusion samples, 31 (23 with FIP, eight controls) showed positive ICC results (Table 4 ). One effusion sample was considered non-diagnostic owing to the absence of cellular material, and this sample was excluded from statistical analysis. Diagnostic sensitivity, specificity, NPV, PPV and overall accuracy are presented in Table 5.

\section{Discussion}

The aim of this study was to determine the usefulness of ICC in effusion as a tool to diagnose FIP. While the demonstration of FCoV antigen in macrophages by immunofluorescence in effusion has previously been assessed, $, 17,22-25$ this study is the first to evaluate the use of ICC analysis in the diagnosis of FIP.

A relatively high number of false-positive test results were identified in this study, consequently leading to a diagnostic specificity of only $72.4 \%$. This is in stark contrast to the results of immunofluorescence assays in older studies that reported a specificity and PPV of $100 \%$ for immunofluorescence testing of effusion. $17,23-25$ However, Litster et al recently reported false-positive results in immunofluorescence in 2/17 cats (specificity $71.4 \%){ }^{22}$ This specificity is very similar to that of the 
Table 2 Cellularity and results of immunostaining of the effusion samples from the 27 cats of the feline infectious peritonitis group

\begin{tabular}{|c|c|c|}
\hline \multirow[t]{2}{*}{ Cat } & \multicolumn{2}{|l|}{ Effusion } \\
\hline & Cellularity ${ }^{\star}$ & Immunostaining $^{\dagger}$ \\
\hline 1 & +++ & +++ \\
\hline 2 & - & ND \\
\hline 3 & +++ & Non-specific $\ddagger$ \\
\hline 4 & +++ & +++ \\
\hline 5 & +++ & +++ \\
\hline 6 & +++ & +++ \\
\hline 7 & +++ & ++ \\
\hline 8 & ++ & + \\
\hline 9 & ++ & +++ \\
\hline 10 & + & - \\
\hline 11 & $\begin{array}{l}\text { Ascites: }++ \\
\text { Pleural effusion: }++\end{array}$ & $\begin{array}{l}\text { Ascites: }++ \\
\text { Pleural effusion: }+\end{array}$ \\
\hline 12 & +++ & + \\
\hline 13 & ++ & + \\
\hline 14 & +++ & + \\
\hline 15 & +++ & +++ \\
\hline 16 & ++ & - \\
\hline 17 & ++ & + \\
\hline 18 & ++ & +++ \\
\hline 19 & ++ & +++ \\
\hline 20 & ++ & ++ \\
\hline 21 & ++ & +++ \\
\hline 22 & + & + \\
\hline 23 & +++ & + \\
\hline 24 & + & + \\
\hline 25 & +++ & + \\
\hline 26 & +++ & + \\
\hline 27 & ++ & Non-specific \\
\hline
\end{tabular}

$*(+)=$ Low cellular $(<3$ cells per high power field [HPF]); $(++)=$ medium cellular $(4-10$ cells per HPF); $(+++)=$ high cellular $(>11$ cells per HPF); $(-)=$ lack of cellular material, non-diagnostic

${ }^{\dagger}(+)=$ Low-yield positive immunostaining (few single macrophages with strong positive signal); $(++)=$ medium-yield positive immunostaining (about half of the macrophages with strong positive signal); $(+++)=$ high-yield positive immunostaining (majority of macrophages with strong positive signal); $(-)=$ negative immunostaining

‡Non-specific staining was defined as diffuse and/or light cytoplasmic staining of macrophages. Samples with non-specific staining were regarded as immunocytochemistry negative

$\mathrm{ND}=$ not determined

present study. It remains unclear why cats without FIP can show positive staining of FCoV antigen in macrophages. Of the eight cats with false-positive ICC results in the present study, three suffered from lymphoma, two had carcinoma and three other cats had decompensated cardiac disease. In four cats (two with lymphoma, one with adenocarcinoma and one with chronic cardiomyopathy), full post-mortem examination, including histopathology and IHC, was performed.
Table 3 Cellularity and results of immunostaining of the effusion samples from the 29 cats of the control group

\begin{tabular}{|c|c|c|}
\hline \multirow[t]{2}{*}{ Cat } & \multicolumn{2}{|l|}{ Effusion } \\
\hline & Cellularity* & Immunostaining ${ }^{\dagger}$ \\
\hline 1 & +++ & - \\
\hline 2 & +++ & - \\
\hline 3 & +++ & Non-specific $M \varphi^{\ddagger}$ \\
\hline 4 & +++ & - \\
\hline 5 & +++ & - \\
\hline 6 & +++ & Non-specific PMNs \\
\hline 7 & +++ & Non-specific MCs \\
\hline 8 & +++ & Non-specific MCs \\
\hline 9 & + & - \\
\hline 10 & +++ & Non-specific PMNs \\
\hline 11 & +++ & - \\
\hline 12 & +++ & +++ \\
\hline 13 & +++ & Non-specific RBCs \\
\hline 14 & ++ & - \\
\hline 15 & +++ & +++ \\
\hline 16 & ++ & Non-specific M $\varphi$ \\
\hline 17 & ++ & Non-specific RBCs \\
\hline 18 & +++ & Non-specific RBCs \\
\hline 19 & +++ & - \\
\hline 20 & ++ & ++ \\
\hline 21 & +++ & - \\
\hline 22 & ++ & ++ \\
\hline 23 & +++ & - \\
\hline 24 & +++ & + \\
\hline 25 & +++ & + \\
\hline 26 & ++ & ++ \\
\hline 27 & + & - \\
\hline 28 & +++ & + \\
\hline 29 & ++ & - \\
\hline
\end{tabular}

$*(+)=$ Low cellular $(<3$ cells per high power field [HPF]); $(++)=$ medium cellular $(4-10$ cells per HPF); $(+++)=$ high cellular $(>11$ cells per HPF); $(-)=$ lack of cellular material, non-diagnostic $\dagger(+)=$ Low-yield positive immunostaining (few single macrophages with strong positive signal); $(++)=$ medium-yield positive immunostaining (about half of the macrophages with strong positive signal); $(+++)=$ high-yield positive immunostaining (majority of macrophages with strong positive signal); $(-)=$ negative immunostaining

‡Non-specific staining was defined as diffuse and/or light cytoplasmic staining of macrophages $(\mathrm{M} \varphi)$ or different cells $(\mathrm{MCs}=$ mesothelial cells; PMNs = polymorphonuclear leukocytes; RBCs = red blood cells). Samples with non-specific staining were regarded as immunocytochemistry negative

In the other four cats (two with decompensated cardiac disease, one with lymphoma and one with carcinoma), the diagnosis was established by cytology to diagnose neoplasia or by echocardiography to diagnose decompensated cardiac disease. It cannot fully be excluded that these eight cats suffered from FIP in addition to their diagnosed diseases. Even in the four cats that had necropsy performed, it might be that they suffered from early-stage FIP, but histopathological lesions were still 
Table 4 Results of immunocytochemistry (ICC) in 56 effusion samples from the 55 cats (one effusion sample was considered non-diagnostic and was therefore excluded from the statistical analysis)

\begin{tabular}{lrcc|} 
& FIP & Control & Total \\
\hline Positive ICC & 23 & 8 & 31 \\
Negative ICC & 4 & 21 & 25 \\
Total & 27 & 29 & 56 \\
\hline
\end{tabular}

FIP = feline infectious peritonitis

Table 5 Sensitivity, specificity, negative predictive value (NPV), positive predictive value (PPV) and overall accuracy of immunocytochemistry (ICC) and prevalence of feline infectious peritonitis in 56 samples from the 55 cats (one effusion sample was considered non-diagnostic and was therefore excluded from the statistical analysis)

\begin{tabular}{ll} 
& ICC in effusion \\
\hline Sensitivity $(95 \% \mathrm{CI})$ & $85.2(66.3-95.8)$ \\
Specificity $(95 \% \mathrm{CI})$ & $72.4(52.8-87.3)$ \\
NPV $(95 \% \mathrm{CI})$ & $84.0(63.9-95.5)$ \\
PPV $(95 \% \mathrm{CI})$ & $74.2(55.4-88.1)$ \\
Overall accuracy $(95 \% \mathrm{CI})$ & $78.6(65.6-88.4)$ \\
Prevalence & 48.2
\end{tabular}

Data are \% (95\% confidence interval)

absent in the examined tissue material. Two of the eight cats with false-positive ICC results (both with decompensated cardiac disease) also presented 'FIP-typical' effusion cytology; and the cytology was at least 'compatible with FIP' in another two cats suffering from lymphoma or adenocarcinoma, respectively. However, in three of them, histopathology was performed, but they did not reveal FIP-typical lesions. If the eight cats of the control group that had positive ICC results actually had FIP, the specificity of the ICC assay might have been falsely reduced.

The target cell for viral replication is the macrophage. ${ }^{10,26-28}$ Thus, it is important to distinguish macrophages from other types of cells and to only consider staining of macrophages as true-positive during microscopic evaluation of the immunostained slides. The binding of the antibody to cellular structures other than FCoV antigen might lead to non-specific staining of other cells (eg, mesothelial cells, neutrophils, red blood cells). It is possible that some of these cells were misclassified as macrophages upon reading the slides. Nevertheless, these cell types usually can easily be differentiated and therefore this seems unlikely.

The antibody used in the ICC protocol in this study is known to be specific for the nucleocapsid of FCoV. Nevertheless, non-specific binding of the antibody to other structures within the cytoplasm of macrophages also might lead to false-positive results.

As all the slides were kept in close proximity during the staining procedure, cross-contamination between the slides cannot completely be ruled out. The inclusion of negative control slides could help rectify this problem in future investigations.

Moreover, the staining of non-mutated $\mathrm{FCoV}$ within macrophages cannot fully be excluded. Monocyteassociated viraemia in healthy cats that never developed FIP has been reported earlier, ${ }^{18,29-32}$ allowing for systemic spread of FECV. ${ }^{33}$ It is possible that cell-bound FECV might reach the body cavities through leakage of blood components, especially in the presence of inflammation. ${ }^{34}$

The diagnostic sensitivity of the ICC was $85.2 \%$ in effusion samples. Similar or even lower sensitivities of $57-95 \%$ have been reported previously for immunofluorescence staining of effusion. ${ }^{17,23-25}$ Recently, however, a sensitivity of $100 \%$ was reported for immunofluorescence staining. ${ }^{22}$ This variation can possibly be explained by differences in the sample collection. In the present study and in some of the former studies mentioned, effusion samples were collected both ante- and post mortem, ${ }^{23,24}$ while the study of Litster et al only included effusion specimens collected ante-mortem. ${ }^{22}$ In the present study, all of the four effusion samples with falsenegative ICC results were obtained post mortem. This could have negatively influenced the sensitivity of the test. Unfortunately, the delay between death and sample collection was not always recorded as some cats were directly submitted for necropsy from referring veterinarians. However, all of the cats with FIP, including those with false-negative ICC results, showed positive IHC results. In addition, it is possible that $\mathrm{FCoV}$ antigen was competitively bound in immune complexes by circulating antibodies and therefore could not be detected by the primary antibody used in the ICC protocol. Finally, a monoclonal antibody was used for ICC staining in this study. It is possible that the sensitivity would have been better had a polyclonal antibody been used. However, previous studies using a polyclonal antibody conjugate in immunofluorescence tests reported even lower sensitivities in effusion. ${ }^{17,25}$

One effusion specimen was considered non-diagnostic owing to the lack of cellular material on the slide. It is possible that the body fluid had a low cell count or the cells were destroyed or washed off during the processing of the slide; the steps, such as cytocentrifugation, freezing, thawing and washing, can decrease the number of adequate cells on the slide. Overall, cellularity was higher on haematoxylin and eosin-stained slides than on immunostained slides. The inclusion of a fixation step in the staining protocol might lead to a better cellularity of the ICC samples and should be further investigated. 
The major study limitation is possible misclassification bias as cats in the control ('FIP-negative') group could possibly have suffered from early-stage FIP in addition to another disease. Some of the control cases yielded positive ICC results; if these cats were misclassified, then the specificity of the ICC test might have been falsely reduced. In addition, if some control cats with negative ICC results truly had FIP, the diagnostic sensitivity would have been falsely increased.

Another limitation is the fact that the slides were stored at $-20^{\circ} \mathrm{C}$ until use. Sample degradation can occur unless specimens are stored at $-80^{\circ} \mathrm{C}$, which might have influenced the diagnostic performance of the ICC assay.

\section{Conclusions}

The present study evaluated the utility of an ICC assay in the diagnosis of FIP using effusion samples. The diagnostic specificity was $72.4 \%$, while diagnostic sensitivity was $85.2 \%$. As specificity is a far more important parameter in a lethal disease like FIP, the usefulness of this method is limited based on the results of the present study, and ICC cannot, in its present form, be used to confirm a suspicion of FIP.

Acknowledgements We would like to thank Karin Stingl, Institute of Veterinary Pathology, for her excellent technical support while performing the ICC and IHC tests.

Conflict of interest The authors declared no potential conflicts of interest with respect to the research, authorship, and/ or publication of this article.

Funding The authors received no financial support for the research, authorship, and/or publication of this article.

Supplementary material Semiquantitative scores empirically established for feline effusions cytospun by centrifuge Universal R (Hettich) at the given settings and using columns with a 27 high power field cross-sectional area.

\section{References}

1 Pedersen N, Boyle J and Floyd K. Infection studies in kittens, using feline infectious peritonitis virus propagated in cell culture. Am J Vet Res 1981; 42: 363-367.

2 Pedersen NC, Boyle JF, Floyd K, et al. An enteric coronavirus infection of cats and its relationship to feline infectious peritonitis. Am J Vet Res 1981; 42: 368-377.

3 Pedersen NC. Virologic and immunologic aspects of feline infectious peritonitis virus infection. Adv Exp Med Biol 1987; 218: 529-550.

4 Compton SR, Barthold SW and Smith AL. The cellular and molecular pathogenesis of coronaviruses. Lab Anim Sci 1993; 43: 15-28.

5 Horzinek M, Herrewegh A and De Groot R. Perspectives on feline coronavirus evolution. Feline Pract 1995; 23: 34-39.

6 Pedersen NC, Liu H, Dodd KA, et al. Significance of coronavirus mutants in feces and diseased tissues of cats suffering from feline infectious peritonitis. Viruses 2009; 1: 166-184.

7 Vennema H, Poland A, Foley J, et al. Feline infectious peritonitis viruses arise by mutation from endemic feline enteric coronaviruses. Virology 1998; 243: 150-157.

8 Poland AM, Vennema H, Foley JE, et al. Two related strains of feline infectious peritonitis virus isolated from immunocompromised cats infected with a feline enteric coronavirus. J Clin Microbiol 1996; 34: 3180-3184.

9 Dewerchin HL, Cornelissen E and Nauwynck HJ. Replication of feline coronaviruses in peripheral blood monocytes. Arch Virol 2005; 150: 2483-2500.

10 Stoddart CA and Scott FW. Intrinsic resistance of feline peritoneal macrophages to coronavirus infection correlates with in vivo virulence. J Virol 1989; 63: 436-440.

11 Rottier PJ, Nakamura K, Schellen P, et al. Acquisition of macrophage tropism during the pathogenesis of feline infectious peritonitis is determined by mutations in the feline coronavirus spike protein. J Virol 2005; 79: 1412214130.

12 Addie D, Belak S, Boucraut-Baralon C, et al. Feline infectious peritonitis. $A B C D$ guidelines on prevention and management. J Feline Med Surg 2009; 11: 594-604.

13 Hartmann K. Feline infectious peritonitis. Vet Clin North Am Small Anim Pract 2005; 35: 39-79.

14 Giori L, Giordano A, Giudice C, et al. Performances of different diagnostic tests for feline infectious peritonitis in challenging clinical cases. J Small Anim Pract 2011; 52: 152-157.

15 Berti-Bock G, Vial F, Premuda L, et al. Exudates, transudates and the Rivalta reaction (1895). Current status and historical premises [article in Italian]. Minerva Med 1979; 70: 3573-3580.

16 Fischer Y, Sauter-Louis C and Hartmann K. Diagnostic accuracy of the Rivalta test for feline infectious peritonitis. Vet Clin Pathol 2012; 41: 558-567.

17 Hartmann K, Binder C, Hirschberger J, et al. Comparison of different tests to diagnose feline infectious peritonitis. J Vet Intern Med 2003; 17: 781-790.

18 Herrewegh AA, de Groot RJ, Cepica A, et al. Detection of feline coronavirus RNA in feces, tissues, and body fluids of naturally infected cats by reverse transcriptase PCR. J Clin Microbiol 1995; 33: 684-689.

19 Kennedy MA, Brenneman K, Millsaps RK, et al. Correlation of genomic detection of feline coronavirus with various diagnostic assays for feline infectious peritonitis. J Vet Diagn Invest 1998; 10: 93-97.

20 Kipar A and Meli ML. Feline infectious peritonitis: still an enigma? Vet Pathol 2014; 51: 505-526.

21 Pedersen NC. An update on feline infectious peritonitis: diagnostics and therapeutics. Vet J 2014; 201: 133-141.

22 Litster AL, Pogranichniy R and Lin TL. Diagnostic utility of a direct immunofluorescence test to detect feline coronavirus antigen in macrophages in effusive feline infectious peritonitis. Vet J 2013; 198: 362-366.

23 Paltrinieri S, Parodi MC and Cammarata G. In vivo diagnosis of feline infectious peritonitis by comparison of protein content, cytology, and direct immunofluorescence test on peritoneal and pleural effusions. J Vet Diagn Invest 1999; 11: 358-361. 
24 Parodi MC, Cammarata G, Paltrinieri S, et al. Using direct immunofluorescence to detect coronaviruses in peritoneal in peritoneal and pleural effusions. J Small Anim Pract 1993; 34: 609-613.

25 Hirschberger J, Hartmann K, Wilhelm N, et al. Clinical symptoms and diagnosis of feline infectious peritonitis [article in German]. Tierarztl Prax 1995; 23: 92-99.

26 Weiss RC and Scott FW. Pathogenesis of feline infectious peritonitis: nature and development of viremia. Am J Vet Res 1981; 42: 382-390.

27 Kipar A, Bellmann S, Kremendahl J, et al. Cellular composition, coronavirus antigen expression and production of specific antibodies in lesions in feline infectious peritonitis. Vet Immunol Immunopathol 1998; 65: 243-257.

28 Kipar A, May H, Menger S, et al. Morphologic features and development of granulomatous vasculitis in feline infectious peritonitis. Vet Pathol 2005; 42: 321-330.

29 Gunn-Moore DA, Gruffydd-Jones TJ and Harbour DA. Detection of feline coronaviruses by culture and reverse transcriptase-polymerase chain reaction of blood samples from healthy cats and cats with clinical feline infectious peritonitis. Vet Microbiol 1998; 62: 193-205.

30 Fehr D, Bolla S, Herrewegh AA, et al. Detection of feline coronavirus using RT PCR: basis for the study of the pathogenesis of feline infectious peritonitis (FIP) [article in German]. Schweiz Arch Tierheilkd 1996; 138: 74-79.

31 Meli M, Kipar A, Muller C, et al. High viral loads despite absence of clinical and pathological findings in cats experimentally infected with feline coronavirus (FCoV) type $I$ and in naturally FCoV-infected cats. J Feline Med Surg 2004; 6: 69-81.

32 Kipar A, Baptiste K, Barth A, et al. Natural FCoV infection: cats with FIP exhibit significantly higher viral loads than healthy infected cats. J Feline Med Surg 2006; 8: 69-72.

33 Kipar A, Meli ML, Baptiste KE, et al. Sites of feline coronavirus persistence in healthy cats. J Gen Virol 2010; 91: 1698-1707.

34 Addie DD, Paltrinieri S and Pedersen NC. Recommendations from workshops of the second international feline coronavirus/feline infectious peritonitis symposium. J Feline Med Surg 2004; 6: 125-130. 